\title{
Jörg Gawlick
}

Die stufenweise Wiedereingliederung

arbeitsunfähiger Arbeitnehmer 


\title{
FORUM ARBEITS- UND SOZIALRECHT
}

\author{
herausgegeben von \\ Prof. Dr. Richard Giesen, Prof. Dr. Matthias Jacobs, \\ Prof. Dr. Dr. h.c. Horst Konzen und Prof. Dr. Meinhard Heinze †
}

Band 31 


\section{Die stufenweise Wiedereingliederung arbeitsunfähiger Arbeitnehmer in das Erwerbsleben nach § 28 SGB IX / § 74 SGB V}

Eine arbeitsrechtliche Betrachtung

Jörg Gawlick

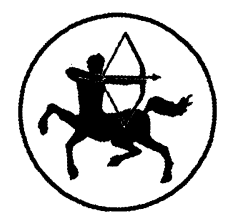

Centaurus Verlag \& Media UG 2008 


\section{Zum Autor:}

Jörg Gawlick, geb. 1977, absolvierte nach seiner Ausbildung zum Bankkaufmann als Stipendiat der Hamburger Sparkasse ein Studium der Rechtswissenschaften an der Universität Hamburg. 2008 promovierte er bei Prof. Dr. Matthias Jacobs an der Bucerius Law School. Derzeit ist er als Rechtsreferendar tätig.

\section{Die Deutsche Bibliothek - Cip-Einheitsaufnahme}

\section{Gawlick, Jörg:}

Die stufenweise Wiedereingliederung arbeitsunfähiger Arbeitnehmer in das Erwerbsleben nach § 28 SGB IX / § 74 StGB V : Eine arbeitsrechtliche Betrachtung / Jörg Gawlick. - Freiburg: Centaurus-Verl., 2009

(Forum Arbeits- und Sozialrecht; Bd. 31)

Zugl.: Hamburg, Bucerius Law School, 2008

ISBN 978-3-8255-0725-1

DOI 10.1007/978-3-86226-380-6

\section{ISSN 0936-028X}

Alle Rechte, insbesondere das Recht der Vervielfältigung und Verbreitung sowie der Übersetzung, vorbehalten. Kein Teil des Werkes darf in irgendeiner Form (durch Fotokopie, Mikrofilm oder ein anderes Verfahren) ohne schriftliche Genehmigung des Verlages reproduziert oder unter Venwendung elektronischer Systeme verarbeitet, vervielfältigt oder verbreitet werden.

C CENTAURUS Verlag \& Media KG, Freiburg 2009

Umschlaggestaltung: Antje Walter, Titisee-Neustadt Satz: Vorlage des Autors 
Meiner Familie 


\section{Vorwort}

Die vorliegende Arbeit wurde im Herbsttrimester 2008 vom Promotionsausschuss der Bucerius Law School - Hochschule für Rechtswissenschaft -, Hamburg, als Dissertation angenommen. Die mündliche Prüfung erfolgte am 16. Dezember 2008. Das Manuskript wurde im Februar 2008 abgeschlossen. Bis zum Oktober 2008 veröffentlichte Literatur und Rechtsprechung wurden nachgetragen.

Mein herzlicher Dank gilt meinem Doktorvater Professor Dr. Matthias Jacobs, der die Arbeit von der ersten Idee bis zur Veröffentlichung in der von ihm herausgegebenen Schriftenreihe intensiv unterstützt und gefördert hat.

Außerdem danke ich Frau Professor Dr. Birgit Weitemeyer für die sehr zügige Erstellung des Zweitgutachtens.

Ferner gilt mein Dank der Hamburger Sparkasse für die finanzielle Unterstützung meines Promotionsvorhabens.

Darüber hinaus danke ich Herrn Dennis Pohl, der die Entstehung der Arbeit mit seiner ständigen Diskussionsbereitschaft und vielen kritischen Hinweisen begleitet hat.

Schließlich schulde ich meiner Familie ganz besonderen Dank für ihren Zuspruch, ihre Geduld und ihre vorbehaltlose Unterstützung. Ihr widme ich diese Arbeit.

Hamburg, im Dezember 2008

Jörg Gawlick 


\section{Inhaltsverzeichnis}

Abkürzungsverzeichnis.

XIX

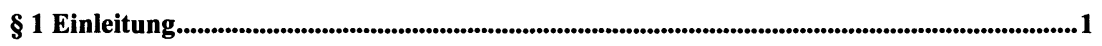

A. Krankheitsbedingte Fehlzeiten sowie ökonomische Überlegungen.....................................1

B. Wiedereingliederung am Beispiel der Ford Werke GmbH .............................................4

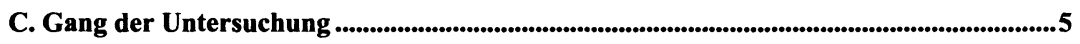

§ 2 Modell, Funktion, Begründung und Rechtsnatur der Wiedereingliederung......................7 7

A. Modell der stufenweisen Wiedereingliederung...............................................................................9

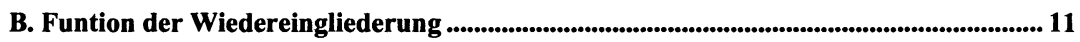

C. Rechtscharakter des Wiedereingliederungsverhältnisses ....................................................... 12

I. Das Wiedereingliederungsverhältnis als Gefälligkeitsverhältnis....................................... 12

1. Gefälligkeitsverhältnisse versus vertragliche Schuldverhältnisse................................... 13

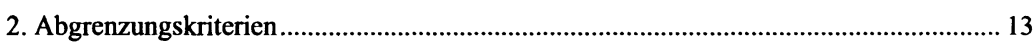

a) Die Kriterien der Uneigennützigkeit und der Interessenlage ...................................... 14

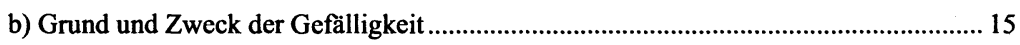

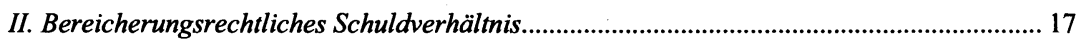

III. Modifiziertes Arbeitsverhältnis oder Rechtsverhältnis eigener Art .................................... 18

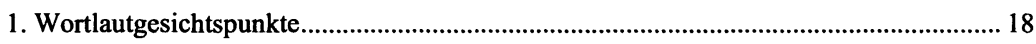

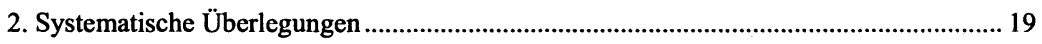

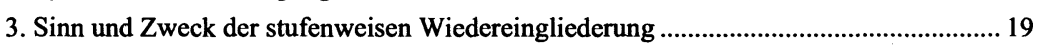

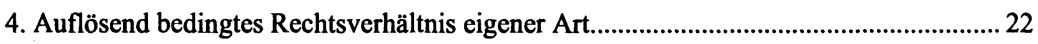

D. Freiwilligkeit der Wiedereingliederung....................................................................................... 23

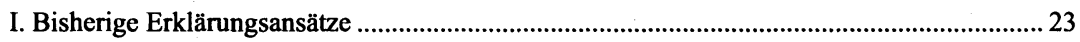

1. Wiedereingliederungsvereinbarung im Belieben der Parteien ....................................... 23

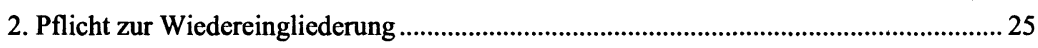

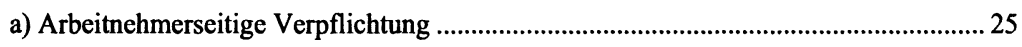

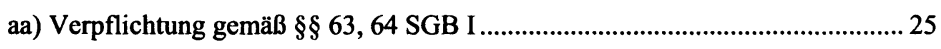

bb) Regelungen des Bürgerlichen Gesetzbuches ................................................. 27 


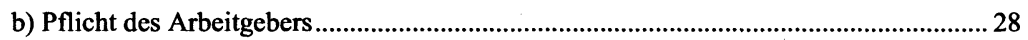

aa) Pflicht durch arbeitsrechtlichen Gleichbehandlungsgrundsatz ....................... 28

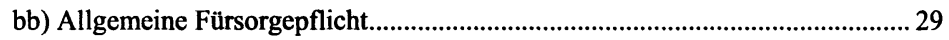

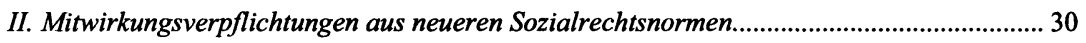

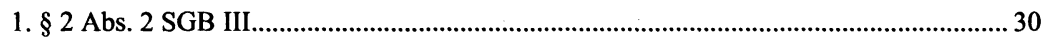

a) Die sozialrechtliche Norm des § 2 SGB III im Arbeitsrecht......................................... 31

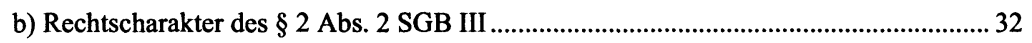

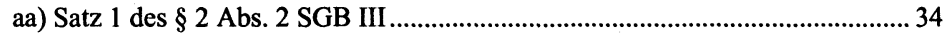

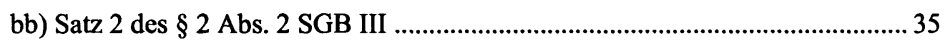

(1) Sollvorschriften als verpflichtende Normen ................................................. 35

(2) Regelbeispiele Nr. 1 und Nr. 2 des $\S 2$ Abs. 2 S. 2 SGB III ......................... 36

(3) Regelbeispiel Nr. 3 des $\S 2$ Abs. 2 S. 2 SGB III............................................. 39

cc) Ergebnis zur rechtlichen Einordnung des $\S 2$ Abs. 2 SGB III ......................... 41

c) Ausstrahlung des $\S 2$ Abs. 2 SGB III auf Wiedereingliederungsfragen .......................... 41

2. $\S 81$ Abs. 4 S. 1 Nr. 1 SGB IX und $\S 84$ Abs. 2 SGB IX ................................................ 43

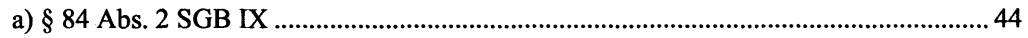

aa) Wiedereingliederungspflicht unmittelbar aus $\S 84$ Abs. 2 SGB IX................. 44

bb) $\S 84$ Abs. 2 SGB IX als Ausprägung der Fürsorgepflicht ................................ 46

b) Schwerbehinderte Rehabilitanden und $\S 81$ Abs. 4 S. 1 Nr. 1 SGB IX.......................... 47

aa) Der Beschäftigungsanspruch schwerbehinderter Arbeitnehmer ...................... 48

(1) Die Rechtsprechung des Bundesarbeitsgerichtes ......................................... 48

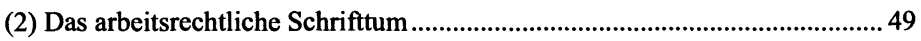

bb) Anspruch auf Wiedereingliederung aus $\S 81$ Abs. 4 S. 1 Nr. 1

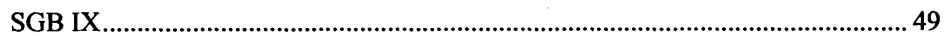

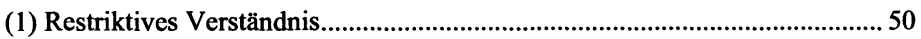

(2) Rekonvaleszenten als Beschäftigte nach § 81 Abs. 4 S. 1 Nr. 1

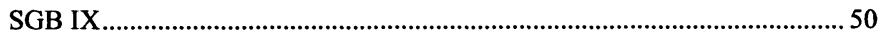

(a) Begriff der Beschäftigung ............................................................................5 50

(b) Von $\S 12$ Abs. 1 SchwbeschG zu $\S 81$ Abs. 4 S. 1 Nr. 1

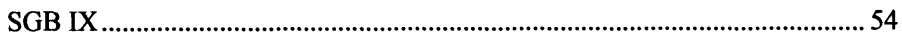

(c) Weitere systematische und teleologische Überlegungen .............................55

cc) Grenzen des Wiedereingliederungsanspruches (§81 Abs. 4 S. 3

SGB IX) 60

c) Nicht schwerbehinderte Rehabilitanden und $\S 81$ Abs. 4 S. 1 Nr. 1

SGB IX 61

E. Zusammenfassung 61 
§ 3 Hauptleistungspflichten/Teilarbeitsunfähigkeit.............................................................6 63

A. Hauptleistungspflichten und Wiedereingliederungen ....................................................... 63

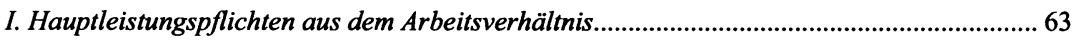

II. Hauptleistungspflichten aus der Wiedereingliederungsmaßnahme ..................................... 64

\section{B. Abgrenzung des Wiedereingliederungsverhältnisses von der}

Teilarbeitsunfähigkeit ........................................................................................................................................ 66

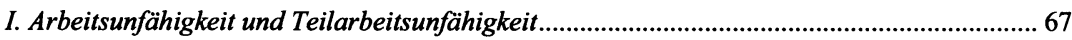

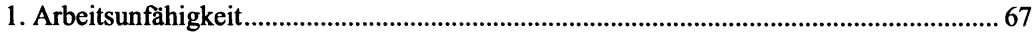

a) Arbeitsrechtlicher Arbeitsunfähigkeitsbegriff ..........................................................67

b) Krankenversicherungsrechtlicher Arbeitsunfähigkeitsbegriff ...................................68

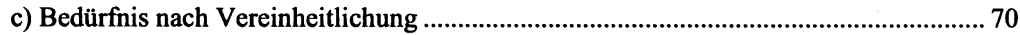

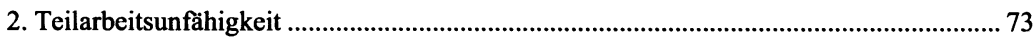

II. Teilarbeitsunfähigkeit im Arbeits- und Sozialrecht ................................................................ 74

1. Referentenentwurf eines Gesetzes zur Förderung der Beschäftigung.............................. 74

2. Ablehnung der Teilarbeitsunfähigkeit und Verneinung jeglicher

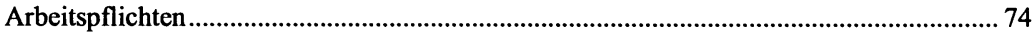

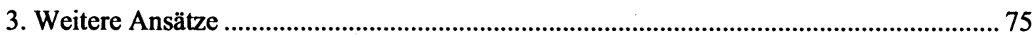

a) Teilarbeitsunfähigkeit als Arbeitsunfähigkeit bei gleichzeitigem

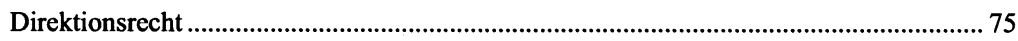

b) Differenzierung zwischen qualitativer und quantitativer

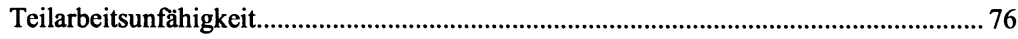

aa) Direktionsrecht nur bei qualitativer Leistungseinschränkung.......................... 76

bb) Anerkennung allein einer quantitativen Teilarbeitsunfähigkeit........................ 77

cc) Bejahung der quantitativen und qualitativen Teilarbeitsunfähigkeit ............... 78

c) Anspruch des Arbeitgebers auf stundenweise Teiltätigkeit ..........................................78

d) Befürwortung einer uneingeschränkten Teilarbeitsunfähigkeit ................................... 79

4. Ablehnung der Teilarbeitsunfähigkeit / Verweisungsmöglichkeit in

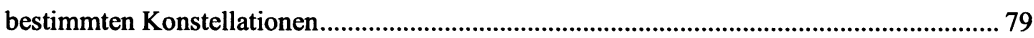

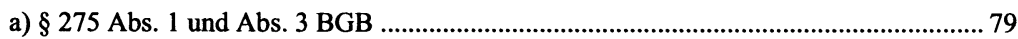

aa) Allgemeines Schuldrecht und quantitative Teilarbeitsunfähigkeit ................... 80

bb) § 275 BGB im Lichte des Schuldrechtsmodernisierungsgesetzes.................... 82

cc) Verhältnis von $\S 275$ Abs. 1 BGB zu $§ 74$ SGB V ........................................ 84

b) Arbeitsunfähigkeit und arbeitgeberseitiges Direktionsrecht .........................................86

aa) Qualitative Einschränkung der Leistungsfähigkeit ........................................8 86

bb) Quantitative Einschränkung der Leistungsfähigkeit....................................... 88

cc) Zusammentreffen von qualitativer und quantitativer

Leistungsbeschränkung....................................................................................... 90

c) Teilarbeitsunfähigkeit, § 74 SGB V und Lastenverteilung im Arbeitsrecht .................. 90

III. Ergebnis zur Abgrenzung der Wiedereingliederung von der Teilarbeitsunfähigkeit.............. 91 
$\S 4$ Wiedereingliederungen und andere Situationen ruhender

Arbeitsverhältnisse

§ 5 Nebenpflichten während der Wiedereingliederung..............................................................97

A. Systematisierung der Nebenpflichten eines Schuldverhältnisses

\section{B. Systematisierung der während einer Wiedereingliederung bestehenden} Nebenpflichten.

C. Auswirkung einer Wiedereingliederung auf die Nebenpflichten aus dem Arbeitsverhältnis

I. Fortdauernde Ausstrahlungswirkung.. 102

II. Notwendigkeit einer Interessenharmonisierung. 102

III. Fortwirkung nur jener Nebenpflichten, die an das Erfüllungsverhältnis anknüpfen.

1. Grund- und Erfüllungsverhältnis als Bestandteile des Arbeitsverhältnisses. 103

2. Differenzierung zwischen Nebenpflichten des Grund- und solchen des

Erfüllungsverhältnisses 106

D. Schicksal einzelner Nebenpflichten aus dem Arbeitsverhältnis in der Wiedereingliederung 107

I. Nebenpflichten des Arbeitnehmers aus dem Arbeitsverhältnis.............................................. 108

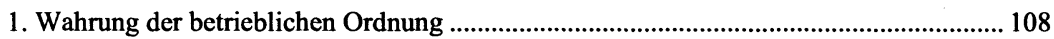

2. Beachtung des Unternehmereigentums, Pflicht zur Schadensabwendung, Anzeigepflicht 109

3. Auskunftspflicht. 110

4. Schmiergeldverbot 111

5. Pflicht zur Verschwiegenheit. 112

6. Wettbewerbesverbot 113

7. Pflicht zu gesundheitsförderndem Verhalten.................................................................. 115

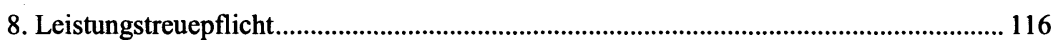

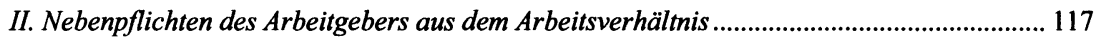

1. Die Beschäftigungspflicht aus dem Arbeitsverhältnis..................................................... 118

2. Aufklärungs- und Informationspflichten ......................................................................... 119

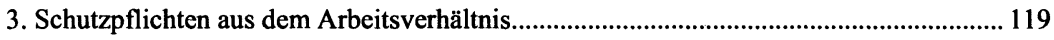

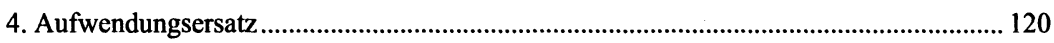


E. Nebenpflichten aus dem Wiedereingliederungsverhältnis

I. Arbeitnehmerseitige Nebenpflichten aus dem Wiedereingliederungsverhältnis

1. Pflicht zu gesundheitsförderndem Verhalten............................................................... 121

2. Pflicht zur Durchführung/Fortsetzung einer Wiedereingliederung................................. 122

3. Schutzpflichten aus dem Wiedereingliederungsverhältnis.............................................. 123

II. Arbeitgeberseitige Nebenpflichten aus dem Wiedereingliederungsverhältnis...................... 124

1. Beschäftigungspflicht aus dem Wiedereingliederungsverhältnis..................................... 124

a) Nicht Schwerbehinderte arbeitsunfähige Arbeitnehmer............................................. 124

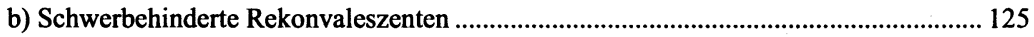

2. Pflicht zur Fortsetzung der Wiedereingliederungsmaßnahme ......................................... 127

3. Schutzpflichten aus dem Wiedereingliederungsverhältnis............................................... 128

F. Konkludent vereinbarte Nebenpflichten im Rahmen der

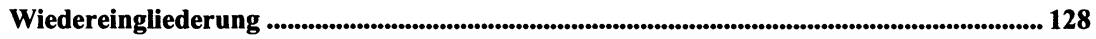

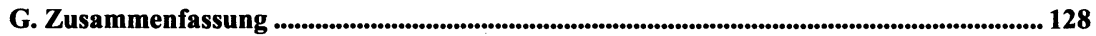

§ 6 Arbeitgeberseitiges Direktionsrecht während der Wiedereingliederung........................... 131

A. Anknüpfungspunkt des auf dem Arbeitsverhältnis beruhendem

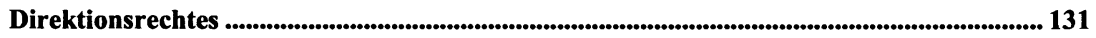

B. Das auf dem Wiedereingliederungsverhältnis beruhende Direktionsrecht.................... 132

C. Zusammenfassung....................................................................................................................................... 134

§ 7 Rechtliche Würdigung von Pflichtverletzungen in Zeiten der stufenweisen

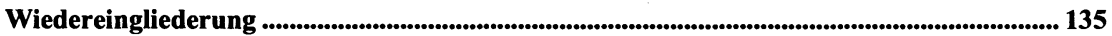

A. Beendigung des Wiedereingliederungs- und/oder des Arbeitsverhältnisses ................... 135

I. Anwendbarkeit des § 1 KSchG auf Wiedereingliederungsverhältnisse ................................... 136

1. $1 \mathrm{KSchG}$ analog und Wiedereingliederungsverhältnisse

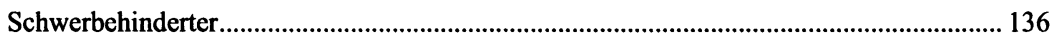

a) Gesetzeslücke als Voraussetzung einer Analogie......................................................... 137

b) Regelungsplan des Kündigungsschutzgesetzes...................................................... 138

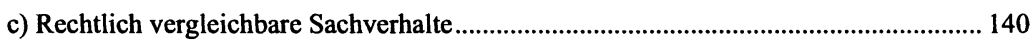

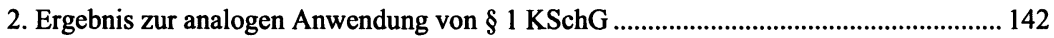

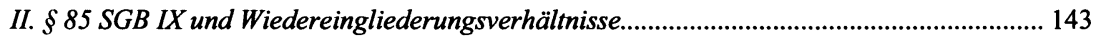

III. Beendigungsmöglichkeiten in Folge von Pflichtverletzungen ......................................... 144

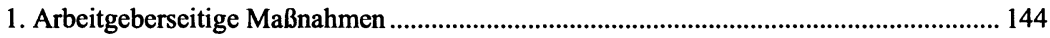

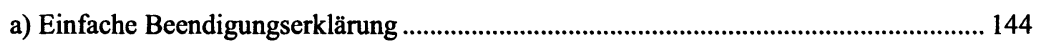




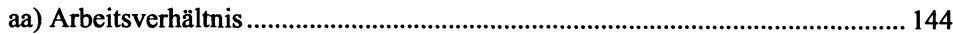

bb) Wiedereingliederungsverhältnis................................................................ 145

(1) Differenzierung zwischen schwerbehinderten und übrigen

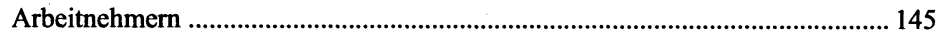

(2) Auslauffrist ................................................................................................. 147

b) Ordentliche und außerordentliche Kündigung im Sinne der $\S \S 620 \mathrm{ff}$.

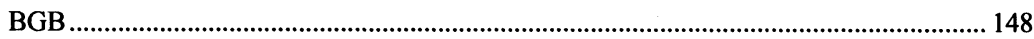

aa) Kündigung des Arbeitsverhältnisses ........................................................... 148

(1) Keine Differenzierung nach dem Ursprung der Nebenpflicht im

Kündigungsfall .................................................................................................. 149

(2) Pflichtverletzung bei Wiedereingliederung als außerdienstliches

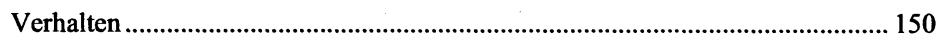

(3) Voraussetzungen der ordentlichen Kündigung bei

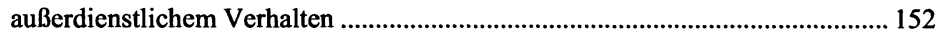

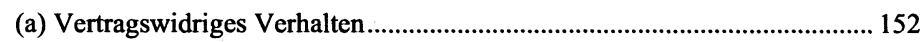

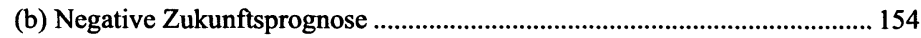

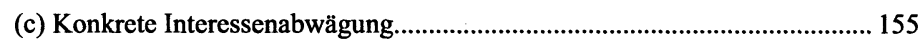

(4) Außerordentliche Kündigung des Arbeitsverhältnisses................................ 157

(a) Allgemeine Voraussetzungen einer Kündigung nach § 626

BGB

(b) Außerdienstliche Pflichtverletzung in Zeiten des ruhenden

Arbeitsverhältnisses ..................................................................................... 158

bb) Die Kündigung des Wiedereingliederungsverhältnisses ................................ 160

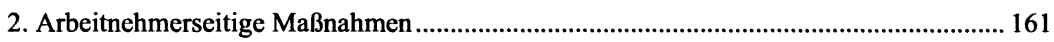

\section{B. Weitere Auswirkungen von Pflichtverletzungen während der}

Wiedereingliederungsphase .....................................................................................................162

I. Erfüllungs- und Unterlassungsansprüche/Klagbarkeit und Durchsetzbarkeit von

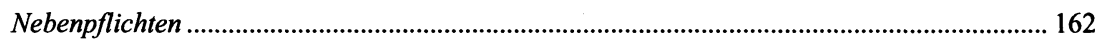

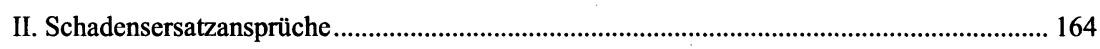

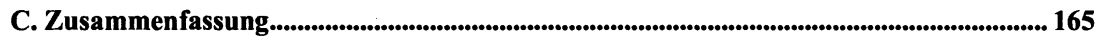

§ 8 Haftungsfragen während der Wiedereingliederung .......................................................... 167

A. Die Arbeitgeberhaftung............................................................................................................................ 168

I. Personenschäden des Arbeitnehmers / Anwendbarkeit des § 104 SGB VII ............................ 168

1. Rehabilitand als Beschäftigter gemäß §§ 7 SGB IV, 2 Abs. 1 Nr. 1 SGB VII ................. 169

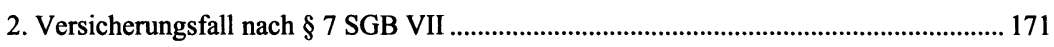

II. Sonstige Schäden des Arbeitnehmers und verschuldensunabhängige

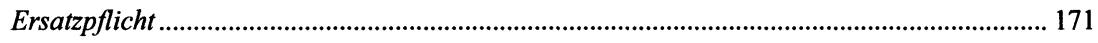


1. §670 BGB analog im vollzogenen Arbeitsverhältnis

B. Die Arbeitnehmerhaftung 174

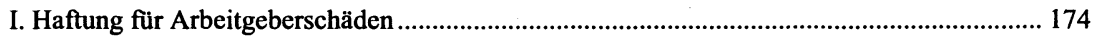

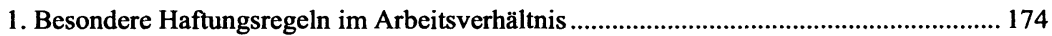

2. Übertragbarkeit der beschränkten Arbeitnehmerhaftung auf das

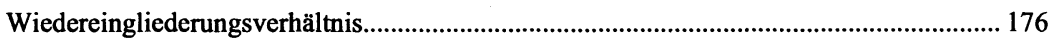

II. Haftung gegenüber Betriebsangehörigen ............................................................................... 179

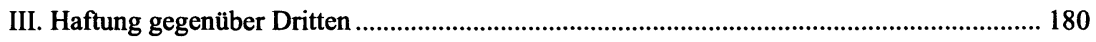

C. Anknüpfung der Haftungsbeschränkungen an das Erfüllungsverhältnis....................... 181

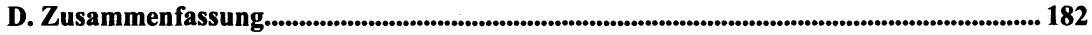

§ 9 Rechtsfolgen und mögliche Maßnahmen bei fehlender Wiedereingliederung ................. 184

A. Individualrechtliche Ebene .......................................................................................................... 184

I. Kündigungsrechtliche Auswirkungen bei unterbliebener Wiedereingliederung.................... 184

1. Kündigungen im Anwendungsbereich des Kündigungsschutzgesetzes............................. 185

a) Unterlassen eines Präventionsverfahrens nach $\S 84$ Abs. 1 und Abs. 2

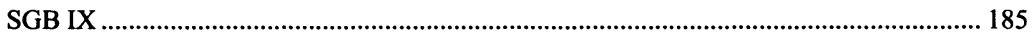

aa) Verhältnis von $\S 84$ Abs. 1 zu $\S 84$ Abs. 2 SGB IX........................................... 185

bb) Wiedereingliederung als Ergebnis einer Maßnahme gemäß $\S 84$

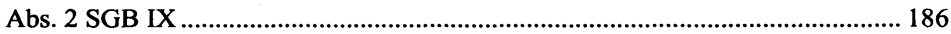

cc) Persönlicher Anwendungsbereich von $\S 84$ Abs. 2 SGB IX............................ 187

dd) $\S 84$ Abs. 2 SGB IX und krankheitsbedingte Kündigung................................. 189

(1) Prüfungskriterien einer krankheitsbedingten Kündigung.............................. 190

(2) Verhältnismäßigkeitsprinzip als Anknüpfungspunkt des

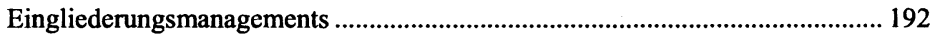

(3) Kündigungsrechtliche Folgen eines unterbliebenen

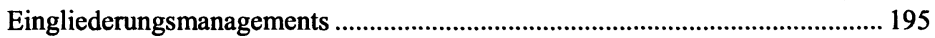

(a) § 84 Abs. 2 SGB IX als Vorschrift ohne jegliche

kündigungsrechtliche Folgen 195

(b) Eingliederungsmanagement als formelle

Wirksamkeitsvoraussetzung

(c) § 84 Abs. 2 SGB IX als Konkretisierung des

Verhältnismäßigkeitsgrundsatzes

(d) Beweisvereitelung bei unterlassener Wiedereingliederung

b) $\S 2$ Abs. 2 S. 2 Nr. 2 SGB III als Bestätigung des

Verhältnismäßigkeitsprinzips 
aa) § 2 Abs. 2 S. 2 Nr. 2 SGB III und Kündigungsfälle ohne

Wiedereingliederungsversuch

bb) Beweisvereitelung bei Verstoß gegen $\S 2$ Abs. 2 S. 2 Nr. 2

SGB III 207

2. Kündigungen außerhalb des Geltungsbereiches des

Kündigungsschutzgesetzes. 209

II. Erfüllungs-/Schadensersatzansprüche des Arbeitnehmers bei verweigerter

Wiedereingliederung. 210

1. Erfüllungsanspruch aus $\S 81$ Abs. 4 S. 1 Nr. 1 SGB IX und Durchsetzbarkeit. 210

a) Erkenntnisverfahren 211

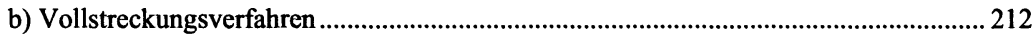

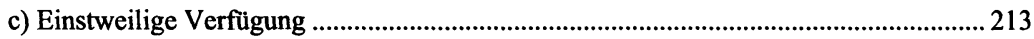

aa) Zutreffende Verfügungsart und ihre Rechtsgrundlage..................................... 214

bb) Voraussetzungen einer Leistungsverfügung ................................................. 216

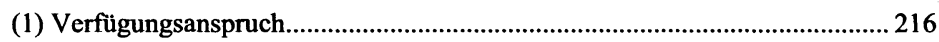

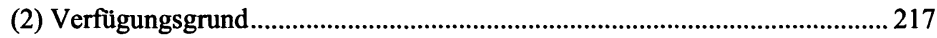

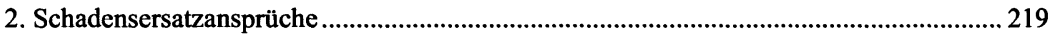

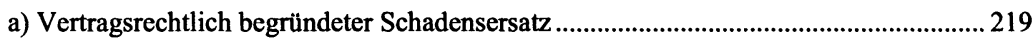

a) Wiedereingliederungsanspruch als Leistungspflicht gemäß

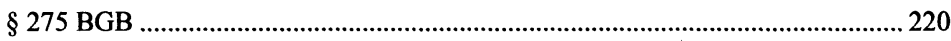

bb) Schadensersatzes statt der Leistung versus einfacher

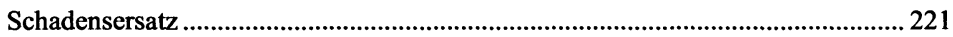

cc) Voraussetzungen eines Anspruches aus $\S \S 280$ Abs. 1 und 3,

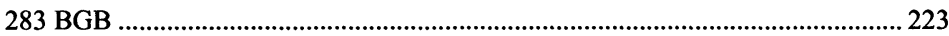

dd) Umfang der Haftung und Naturalherstellung bei Schadensersatz

statt der Leistung........................................................................................................ 224

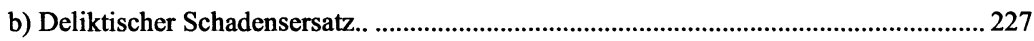

aa) Schadensersatzanspruch aus $\S 823$ Abs. 1 BGB ............................................... 227

bb) Schadensersatzanspruch aus $\S 823$ Abs. 2 BGB.............................................. 227

(1) $\S 81$ Abs. 4 S. 1 Nr. 1 SGB IX als Schutzgesetz nach $\S 823$

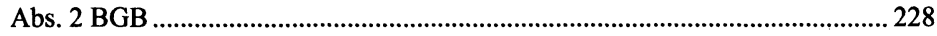

(2) Schadensersatz nach $\S 823$ Abs. 2 BGB in Verbindung mit $\S 84$

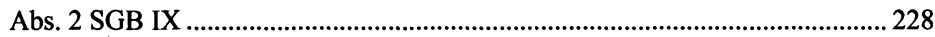

(3) $\S 2$ Abs. 2 SGB III als Schutzgesetz im Sinne des $\S 823$

Abs. 2 BGB

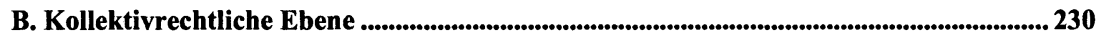

III. Integrationsteam der Ford Werk als Mittel zur Vermeidung

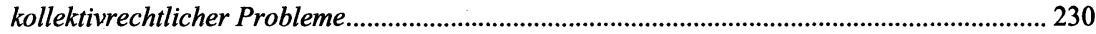

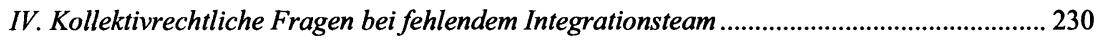


1. Initiativrecht der Arbeitnehmervertretungen hinsichtlich des

Eingliederungsmanagements

2. Erzwingbares Mitbestimmungsrecht bezüglich der Art des

Eingliederungsmanagements

a) Mitbestimmungsrecht des Betriebsrates aus $\S 87$ Abs. 1 Nr. 1 und

Nr. 7 BetrVG

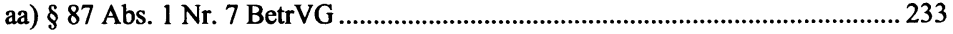

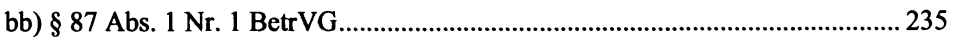

b) Mitbestimmungsrecht der Schwerbehindertenvertretung ..........................................2. 237

3. Rechte der Arbeitnehmervertretungen im Zusammenhang mit einer

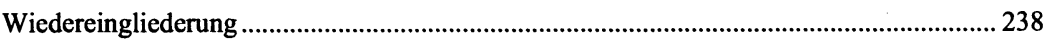

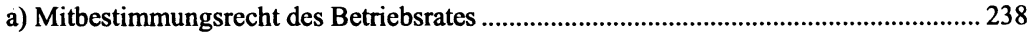

aa) Anwendbarkeit des BetrVG auf Wiedereingliederungsverhältnisse................ 239

(1) Wiedereinzugliedernde als Arbeitnehmer nach §5 Abs. 1

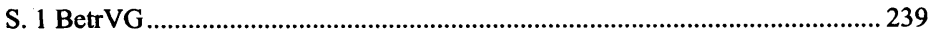

(a) Ruhende Arbeitsverhältnisse von Wehrdienstleistenden ............................ 239

(b) Ruhende Arbeitsverhältnisse von Rehabilitanden ..................................... 240

(2) Wiedereinzugliedernde als Personen gemäß § 5 Abs. 2

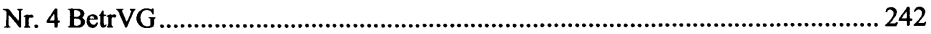

bb) Mitbestimmungsrechte bei Durchführung und Ausgestaltung ........................ 244

b) Mitbestimmungsrecht der Schwerbehindertenvertretung ...........................................245

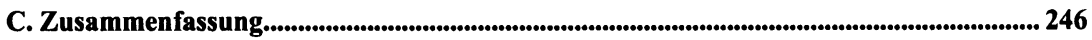

\section{§ 10 Tarifverträge, Betriebsvereinbarungen, § 102 BetrVG und}

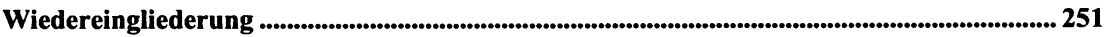

A. Schicksal tarifvertraglicher Regelungen während der

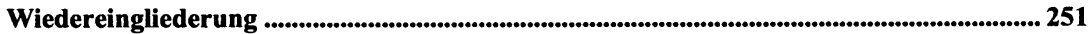

I. Anwendbarkeit von Tarifverträgen auf Wiedereingliederungsverhältnisse ........................... 251

1. Direkte Anwendung von Tarifverträgen ...............................................................................2 251

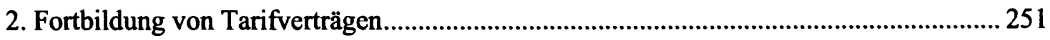

II. Bestimmung fortgeltender Tarifnormen durch Zuordnung zum Grund-oder

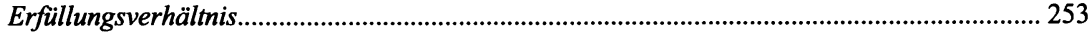

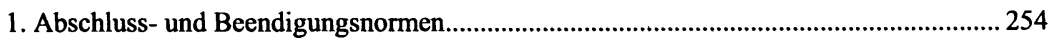

2. Betriebliche und betriebsverfassungsrechtliche Tarifnormen .........................................25

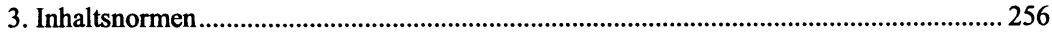


B. Geltung von Betriebsvereinbarungen in Zeiten der Wiedereingliederung....................... 257

C. Anhörungsrecht des Betriebsrates aus § 102 BetrVG .................................................... 258

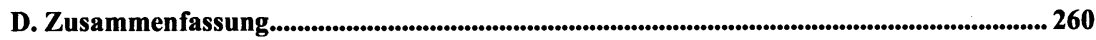

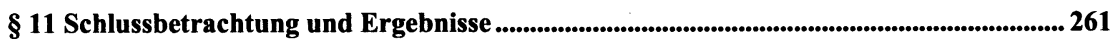

Literaturverzeichnis ........................................................................................................................... 269 


\section{Abkürzungsverzeichnis}

Abs.

AcP

a. F.

AG

AGG

AiB

AP.

AR-Blattei-ES

AR-Blattei-SD

ArbGG.

ArbPISchG.

ArbSchG

ArbuR

Art.

$\mathrm{AuA}$

AU-Richtlinien

BAG.

BB

BetrVG

BGB...

BGB1

Bl.

BPersVG.

BSHG

BT-Drucksache

BUrlG

Absatz

Archiv für die civilistische Praxis (Zeitschrift)

Alte Fassung

Arbeitsgericht

Allgemeines Gleichbehandlungsgestz

Arbeitsrecht im Betreib (Zeitschrift)

Arbeitsrechtliche Praxis

Arbeitsrecht-Blattei Entscheidungssammlung

Arbeitsrecht-Blattei Systematische Darstellungen

Arbeitsgerichtsgesetz

Arbeitsplatzschutzgesetz

Arbeitsschutzgesetz

Arbeit und Recht (Zeitschrift)

Artikel

Arbeit und Arbeitsrecht (Zeitschrift)

Arbeitsunfähigkeits-Richtlinien

Bundesarbeitsgericht

Betriebs-Berater (Zeitschrift)

Betriebsverfassungsgesetz

Bürgerliches Gesetzbuch

Bundesgesetzblatt

Blatt

Bundespersonalvertretungsgesetz

Bundessozialhilfegesetz

Bundestagsdrucksache

Mindesturlaubsgesetz für Arbeitnehmer (Bundesurlaubsgesetz)

BVerfG

Bundesverfassungsgericht

bzw.

Beziehungsweise

DB

Der Betrieb (Zeitschrift)

EFZG.

Entgeltfortzahlungsgesetz

EGBGB....

Einführungsgesetz zum Bürgerlichen Gesetzbuche

ErsK

Die Ersatzkasse (Zeitschrift)

FA

Fachanwalt Arbeitsrecht (Zeitschrift)

f./ff.

folgende/fortfolgende

GewO

Gewerbeordnung 


\begin{tabular}{|c|c|}
\hline G.................. & Grundgesetz \\
\hline HGB................. & Handelsgesetzbuch \\
\hline Hrsg. ................. & Herausgeber \\
\hline HS................... & Halbsatz \\
\hline i. V.m. .............. & In Verbindung mit \\
\hline Jura................. & Juristische Ausbildung (Zeitschrift) \\
\hline jurisPR-ArbR....... & Juris PraxisReport Arbeitsrecht \\
\hline JuS.................. & Juristische Schulung (Zeitschrift) \\
\hline $\mathrm{JZ} \ldots$ & Juristen Zeitung (Zeitschrift) \\
\hline KSchG.............. & Kündigungsschutzgesetz \\
\hline LAG................. & Landesarbeitsgericht \\
\hline LFZG.............. & Lohnfortzahlungsgesetz \\
\hline MDK................ & Medizinischer Dienst der Krankenversicherung \\
\hline MDR................ & Monatszeitschrift für Deutsches Recht (Zeitschrift) \\
\hline MuSchG............ & $\begin{array}{l}\text { Gesetz zum Schutze der erwerbstätigen Mütter } \\
\text { (Mutterschutzgesetz) }\end{array}$ \\
\hline n. F. ............... & Neue Fassung \\
\hline NJW................ & Neue Juristische Wochenschrift (Zeitschrift) \\
\hline NJW-Spezial......... & Neue Juristische Wochenschrift Spezial (Zeitschrift) \\
\hline Nr. ................ & Nummer \\
\hline NZA...$\ldots \ldots \ldots \ldots \ldots$ & Neue Zeitschrift für Arbeitsrecht (Zeitschrift) \\
\hline NZA-RR............ & $\begin{array}{l}\text { Rechtsprechungsreport der Neuen Zeitschrift für Arbeitsrecht } \\
\text { (Zeitschrift) }\end{array}$ \\
\hline PersR................. & Der Personalrat (Zeitschrift) \\
\hline $\operatorname{RdA} \ldots \ldots \ldots \ldots \ldots . . . .$. & Recht der Arbeit (Zeitschrift) \\
\hline Rn. ................. & Randnummer \\
\hline RVO................. & Reichsversicherungsordnung \\
\hline S. ................... & Satz \\
\hline SAE ................ & Sammlung Arbeitsrechtlicher Entscheidungen (Zeitschrift) \\
\hline SchwbBAG......... & $\begin{array}{l}\text { Gesetz zur Bekämpfung der Arbeitslosigkeit Schwerbehin- } \\
\text { derter }\end{array}$ \\
\hline SchwbeschG......... & Gesetz über die Beschäftigung Schwerbeschädigter \\
\hline SchwbG.............. & $\begin{array}{l}\text { Gesetz zur Sicherung der Eingliederung Schwerbehinderter } \\
\text { in Arbeit, Beruf und Gesellschaft (1986) }\end{array}$ \\
\hline SchwbG $1974 \ldots . . .$. & $\begin{array}{l}\text { Gesetz zur Sicherung der Eingliederung Schwerbehinderter } \\
\text { in Arbeit, Beruf und Gesellschaft (1974) }\end{array}$ \\
\hline SGb..... & Die Sozialgerichtsbarkeit (Zeitschrift) \\
\hline SGB I..... & Sozialgesetzbuch (SGB). Erstes Buch (I) - Allgemeiner Teil \\
\hline SGB III.... & $\begin{array}{l}\text { Sozialgesetzbuch (SGB). Drittes Buch (III) - Arbeits- } \\
\text { förderung }\end{array}$ \\
\hline
\end{tabular}


SGB IV

Sozialgesetzbuch (SGB). Viertes Buch (IV) - Gemeinsame Vorschriften für die Sozialversicherung

SGB V............. Sozialgesetzbuch (SGB). Fünftes Buch (V) - Gesetzliche Krankenversicherung

SGB VII........... Sozialgesetzbuch (SGB). Siebtes Buch (VII) - Gesetzliche Unfallversicherung

SGB IX............. Sozialgesetzbuch (SGB). Neuntes Buch (IX) - Rehabilitation und Teilhabe behinderter Menschen

SozSich............. Soziale Sicherheit (Zeitschrift)

SozVers............ Die Sozialversicherung (Zeitschrift)

TVG................ Tarifvertragsgesetz

Urt. ................. Urteil

v. ................... Vom

VersR.............. Versicherungsrecht (Zeitschrift)

vgl. ................ Vergleiche

ZfA............... Zeitschrift für Arbeitsrecht (Zeitschrift)

ZFSH/SGB......... Zeitschrift für Sozialhilfe und Sozialgesetzbuch (Zeitschrift)

ZGS.............. Zeitschrift für das gesamte Schuldrecht (Zeitschrift)

ZIP............... Zeitschrift für Wirtschaftsrecht (Zeitschrift)

zit. ................. Zitiert

ZPO............... Zivilprozessordnung

ZTR............... Zeitschrift für Tarifrecht (Zeitschrift)

ZZP.............. Zeitschrift für Zivilprozess (Zeitschrift) 